\title{
PATHOPHYSIOLOGY, CLINICAL FEATURES AND TREATMENT OF MICROVASCULAR ANGINA: A REVIEW
}

\author{
Hasan Selçuk Özkan ${ }^{1}$, Meral Kayıkçığglu \\ ${ }^{1}$ Ege University School of Medicine, Izmir, TURKEY \\ ${ }^{2}$ Department of Cardiology, Ege University School of Medicine, Izmir, TURKEY
}

\begin{abstract}
Microvascular angina is a non-obstructive coronary syndrome which presents itself as anginal chest pain with different features compared to other causes of angina pectoris. Although, it occurs through a similar mechanism with the better-understood variant (prinzmetal) angina, unlike the former, microvascular angina affects the subendocardial thin, therefore less compliant vessels. It is essential for every medical practitioner to recognize a patient with microvascular angina, as the disease has some characteristic presentations, does not respond well to the classical treatments for angina pectoris, and eventually may result in serious complications later. Keywords: Microvascular angina, coronary syndrome, angina pectoris
\end{abstract}

\section{INTRODUCTION}

Angina pectoris, derived from the Latin verb "angere" (to strangle) and the noun "pectus" (chest), literally meaning strangling of the chest, is chest pain, clinically classified as either typical or atypical by whether special characteristics are present or not. This classification is based on three factors: presence of a substernal pain or discomfort that occurs after exercise or emotional stress, relief by rest or nitroglycerin (1). If all three criteria are present, the pain is classified as a "typical" angina pectoris according to the American College of Cardiology/American Heart Institute's 2002 Guideline Update on Exercise Testing (2). Being an important symptom, angina pectoris may be an indicator of many cardiac pathologies as well as imitated by other causes of chest pain such as those including the gastrointestinal tract, especially esophagus (3).

One of the causes of angina pectoris is microvascular angina (MVA). Acting with a similar mechanism to variant (prinzmetal) angina, MVA is an important condition, mostly considered benign, however responsible for 1.5 times increase in mortality of the affected patients. In addition, patients with MVA tend to have increased pain sensitivity due to the increased adren- ergic and decreased parasympathetic activity; therefore more susceptible to the feeling of pain than other myocardial ischemia patients (4). For all these reasons, patients who show signs of MVA should be recognized by all the physicians and should not be mistaken as extracardiac causes of chest pain $(3,5)$.

\section{PATHOPHYSIOLOGY}

The most common cause of angina pectoris is the lack of oxygen supply through coronaries despite increased demand of the heart tissue. This results in ischemic changes in the area that is supplied by those specific coronary arteries. The main reason behind this lack of supply is the occlusion of the coronary vessels through atherosclerotic changes which is named as coronary artery disease (CAD) (6).

Coronary arteries, give branches reaching to the inner parts of the heart, which are in the proximity of lumens. First branch of these arteries is named, as subepicardial arteries, while they are in the close proximity of the epicardium. The latest branches, on the other hand, are named subendocardial arteries as they are closer to the subendocardium, just beneath the lumen of atria and ventricles (Figure 1). 
The subendocardial layer of the heart is more vulnerable to ischemic changes than subendocardial vessels, which are more compliant as they are thinner. Hence as suggested by the foundational law of compliance, they are more likely to collapse after negative volume changes.

It's well understood that a flow redistribution with a decrease in the flow rate from subendocardium to subepicardium occurs with each myocardial contraction, and the more compliant vessels of the subendocardium induce a greater resistance to the flow (7).

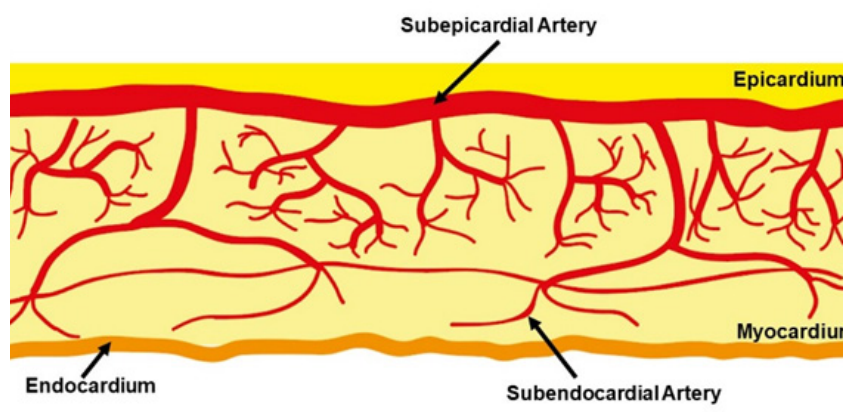

Figure 1: Branching of subepicardial vessels to the subendocardium.

On the other hand, there's another mechanism that can result in decreased perfusion by increasing the resistance against blood flow: abnormal constriction or decreased vasodilation of a coronary artery.

This mechanism is currently the main reason behind MVA (8). However, these erroneous vasodilative properties occur due to a phenomenon known as coronary microvascular dysfunction (CMD) and it is associated with different levels of both vasoactive (nitric oxide) and vasoconstrictive (endothelin-1) substances in different situations.

Coronary microvascular dysfunction can be further divided to 5 subtypes. Type 1, also known as primary CMD occurs in the absence of any previous myocardial disease or epicardial obstruction. Type 2 accompanies myocardial pathologies such as hypertrophic cardiomyopathy. Type 3 occurs in the case of an obstructed coronary artery and Type 4 is iatrogenic and secondary to myocardial revascularization. Finally, Type 5 follows cardiac transplantation (9).

Coronary microvascular dysfunction in MVA seems to appear alone in most cases, therefore can be named as CMD Type 1. Patients with other CMD types are diagnosed more rare than Type 1 (10).

Coronary microvascular dysfunction is a presentation of endothelial cell dysfunction that is associated with hypertension, diabetes mellitus, smoking, hyper- cholesterolemia and with elevated levels of homocysteine.

Homocysteine is known to inhibit vasodilation substances of endothelium such as nitric oxide. Methylenetetrahydrofolate reductase mutations, an enzyme responsible for break down of homocysteine, are also identified in MVA patients (11).

Among the other things, insulin-resistant or insulin-deficient states are also related to endothelial dysfunction, so can contribute to the pathophysiology of MVA (12).

The increased diastolic time is the backbone of another potential mechanism. Gender plays a significant role in this mechanism, older women whose arteries are stiffened tend to have left ventricular hypertrophy resulting in both decreased pressure and time of diastole to supply the coronary flow. It is already known that a decrease in diastolic duration from 33 to $27 \mathrm{~s} /$ min can have the same effect as an increase in coronary stenosis from $40 \%$ to $90 \%$ (13).

Histopathological features of MVA are mainly thickened small arteriolar walls due to medial layer hypertrophy, intimal proliferation and shorter small vessels (14).

\section{CLINICAL FEATURES}

Microvascular angina has a clinical presentation of anginal chest pain, normal or almost-normal epicardial coronary angiography without any spasms and myocardial ischemia in noninvasive stress tests.

Systemic hypertension with or without left ventricular hypertrophy, atypical CAD presentation with diabetes mellitus and as previously stated also from extracardiac causes of chest pain, especially those involving esophagus is a necessity for the specific diagnosis (15). Another common cause of coronary spasm is variant angina (prinzmetal angina). Variant angina patients are usually present with normal coronary angiography although the disease affects subepicardial vessels, and tend to have lower cholesterol and triglyceride levels compared to MVA patients. Additionally, MVA patients have a decreased endothelium-dependent vasodilation of brachial artery after application of a vasodilator compared to the variant angina patients, which suggests a systemic rather than local dysfunction of microvascular bed (2).

Chest pain in MVA is similar to obstructive CAD, mimicking the radiation to the medial part of left arm and substernal compressing pain. However, it occurs at rest in many patients unlike typical angina and can 
last hours and does not respond to nitroglycerin as well as in the typical angina. It is not known to cause systolic ventricular dysfunction (can be determined by a decrease in ejection fraction) as common as it does in CAD. The phenomenon behind this is the layer-selective nature of CMD which only affects the thin layers of the myocardium, thin enough to be supplied also by thin vessels, and does not cause significant tissue damage as in CAD (10).

The electrocardiography (ECG) in MVA is similar to $\mathrm{CAD}$, and shows significant changes in ST-segment such as depression, rarely an elevation during stress. An ambulatory 24-hour-ECG is also no different than chronic stable angina occurring in CAD with circadian episodes of ST-segment depression. ST-segment changes occur in a prolonged manner in MVA than $\mathrm{CAD}$, and mostly accompanied by tachycardia in or- der to increase blood flow through narrowed arteries. The main characteristics of MVA can be summarized as atypical anginal features (long episodes of chest pain lasting sometimes hours and poor nitrate response), exercise-induced chest pain due to the ineffectiveness of physiological vasodilatory mechanisms and pain occurring even at rest, there is no significant systolic ventricular dysfunction present and rare myocardial ischemia can be seen afterward. MVA is more common in women with estrogen deficiency and women have a poorly defined ECG stress test response (15). Hence, a post-menauposal female patient, complaining of prolonged atypical angina pectoris or angina pectoris at rest, should be considered for MVA in the differential diagnosis.

Table 1 compares different causes of angina pectoris by pathophysiology, ECG changes and clinical features.

Table 1: Different characteristics of causes of angina pectoris.

\begin{tabular}{|c|c|c|c|}
\hline Causes of Angina Pectoris & Affected Vessels & ECG Changes & Clinical Features \\
\hline Microvascular Angina & Subendocardial vessels & $\begin{array}{l}\text { Circadian episodes of ST- } \\
\text { segment changes } \\
\text { (elevation or depression) }\end{array}$ & $\begin{array}{l}\text { Prolonged chest pain of } \\
\text { angina characteristics } \\
\text { which may occur both at } \\
\text { rest or exertion, lasting } \\
\text { hours to days and responds } \\
\text { poorly to nitrates, } \\
\text { accompanied by } \\
\text { tachycardia }\end{array}$ \\
\hline $\begin{array}{c}\text { Variant (Prinzmetal) } \\
\text { Angina }\end{array}$ & Subepicardial vessels & $\begin{array}{l}\text { Transient ST-segment } \\
\text { elevation }(\geq 0.1 \mathrm{mV})\end{array}$ & $\begin{array}{l}\text { Chest pain of anginal } \\
\text { characteristics, poor } \\
\text { response to nitrates and } \\
\text { occurs at rest }\end{array}$ \\
\hline Coronary Artery Disease & $\begin{array}{l}\text { Every vessel in coronary } \\
\text { circulation, mostly } \\
\text { affecting larger vessels }\end{array}$ & $\begin{array}{l}\text { Permanent ST-segment } \\
\text { changes, mostly ST- } \\
\text { segment depression (in } \\
\text { chest leads } \geq 0.2 \mathrm{mV} \text {, in } \\
\text { extremity leads } \geq 0.1 \mathrm{mV} \text { ) }\end{array}$ & $\begin{array}{l}\text { Chest pain of anginal } \\
\text { characteristics, good } \\
\text { response to nitrates, occurs } \\
\text { at rest or stress, often last } \\
\text { minutes }\end{array}$ \\
\hline
\end{tabular}




\section{DIAGNOSTICS}

Although there are studies with non-invasive tests to assess indirect myocardial resistance to perfusion during stress, typically administering a systemic vasodilator such as adenosine, the perfusion assessment is not sensitive enough to determine if the problem occurs in the epicardial layer or subendocardial layer.

Invasive coronary angiography can provide valuable information by guide wire-based assessment of the blood flow through coronaries at rest (at the time, where the vessels are vasodilated) and also by using pharmacological such as acetylcholine to mimic vasoconstriction.

The preferred vessel is the left descending coronary artery for this procedure because of its great impact on the perfusion of the myocardium. Multiple vessels can be interrogated but as the duration of the process gets longer the risk increases as well (16).

Nonetheless, in order to perform an invasive procedure such as coronary angiography the patient should exhibit the clinical presentations of angina. If the patient fits the MVA diagnosis criteria with atypical anginal features (long duration, poor response, occurring at rest) and presents with normal epicardial vessels in angiography but only with CMD alone and no other comorbidities, as in most of the cases, should be diagnosed as primary MVA accompanied by CMD Type 1 . CMD can be detected by following angiographical parameters: impaired coronary flow reserve $(\leq 2.0)$, coronary microvascular spasm (not accompanied by epicardial spasm after acetylcholine stimulation), abnormal coronary microvascular resistance indices (Index of Microcirculatory Resistance $>25$ ) and coronary slow flow phenomenon (TIMI frame count $>25$ ) (17).

In addition to the etiologies behind, $\mathrm{CMD}$ can also be classified as either structural CMD (SCMD) or functional CMD (FCMD). FCMD is characterized by low vascular tone both at rest and exercise, and can be recognized with increased nitric oxide synthetase (NOS) activity, normal forearm acetylcholine dilatation (FAD) during angiography and normal exercise blood pressure (EBP) and decreased myocardial coronary perfusion efficiency and slightly elevated N-terminal pro-brain natriuretic peptide (NT-proBNP) levels. SCMD occurs due to high vascular tone both at rest and exercise, and can be recognized with increased NOS activity, however with reduced FAD and higher EBP levels and significantly increased levels of NT-proBNP and a decrease in myocardial coronary perfusion efficiency (18). Positron emission tomogra- phy with myocardial blood flow quantification can also be used to detect microvascular dysfunction with less invasive diagnostic tool, which needs further studies (19).

\section{TREATMENT}

Conventional anti-anginal drugs (nitroglycerine, isosorbide dinitrate, isosorbide mononitrate and erythrityl tetranitrate etc.) are proven to be poorly effective against MVA therefore a different approach should be applied to these patients.

As first-line treatment, beta-blockers are shown to be effective especially with patients of effort angina with increased heart rate (HR) on effort or patients with symptoms of increased adrenergic activity such as increased HR at rest. Ivabradine, a drug acting on If channels mostly located on the sinoatrial node, therefore used to induce bradycardia can be used but there is no evidence supporting its effectivity. To the patients with angina at rest, calcium channel blockers of non-dihydropyridine type, mostly preferred for their anti-arrhythmic properties in other settings, such as diltiazem and verapamil should be effective. Although, oral nitrates are proven to be not effective, nicorandil, a potassium channel activator shown to be effective in some studies, however another agent, ranolazine that reduces late sodium current is promising. In addition to all these, angiotensinogen converter enzyme inhibitors diminishing the oxidant feature of angiotensinogen II and decreasing microvascular dysfunction and alpha blockers, which interfere with adrenergic stimuli can be second-line treatment options. Also, statins are proven to be effective by improving the microvascular dysfunction $(8,20)$. In post-menopausal women, estrogens can also be a part of the treatment (10).

\section{CONCLUSION}

Microvascular angina as an insidious condition, mimicking many other similar etiologies, can easily be overseen, thus falsely diagnosed and managed by physicians. MVA patients are diagnosed mostly with extracardiac causes of chest pain or receive the classical antianginal drugs such as nitroglycerin. However, as the antianginals are poorly effective against MVA, the patient continues to suffer and due to the increased adrenergic stimulation the patient tends to feel the pain intensely. The increased mortality rate is another proof of the importance of this condition (4). Therefore, rec- 
ognition of a patient with MVA by any physician is of utmost importance, just not to relieve the pain of the patient but also to avoid the possible follow-up comorbidities and mortality.

\section{Ethics Committee Approval: N/A}

Informed Consent: N/A

Conflict of Interest: The authors declared no conflict of interest. Author contributions: Concept: HSO, MK Design: HSO, MK Supervision: HSO, MK Resources: HSO, MK Materials: HSO, MK Data collection and/or processing HSO, MK Analysis and/or Interpretation: HSO, MK Literature Search: HSO, MK Writing Manuscript: HSO, MK Critical Review: HSO, MK

Financial disclosure: The authors declared that this study received no financial support.

\section{REFERENCES}

1. Gandhi MM, Lampe FC, Wood DA. Incidence, clinical characteristics, and short-term prognosis of angina pectoris. Heart 1995;73(2):193-8.

2. Hermann LK, Weingart SD, Yoon YM, et al. Comparison of frequency of inducible myocardial ischemia in patients presenting to emergency department with typical versus atypical or nonanginal chest pain. Am J Cardiol 2010;105(11):1561-4.

3. Baggiano A, Guglielmo M, Muscogiuri G et al. (Epicardial and microvascular) angina or atypical chest pain: differential diagnoses with cardiovascular magnetic resonance. Eur Hear J Suppl 2020;22:116-30.

4. Park JJ, Park SJ, Choi DJ. Microvascular angina: Angina that predominantly affects women. Korean J Intern Med 2015;30(2):140-7. 5. Bøtker HE, Sonne HS, Sørensen KE. Frequency of systemic microvascular dysfunction in syndrome $\mathrm{x}$ and in variant angina. Am J Cardiol 1996;78(2):182-6.

6. Dessalvi CC, Deidda M, Giorgi M et al. Vascular damage - coronary artery disease. J Cardiovasc Echogr 2020;30:11-6.

7. Algranati D, Kassab GS, Lanir Y. Why is the subendocardium more vulnerable to ischemia? A new paradigm. Am J Physiol - Hear Circ Physiol 2011;300(3):1090-100.

8. Kayikcioglu M, Payzin S, Yavuzgil O et al. Benefits of statin treatment in cardiac syndrome-X1. Eur Heart J 2003;24(22):1999-2005. 9. Ford TJ, Corcoran D, Berry C. Stable coronary syndromes: Pathophysiology, diagnostic advances and therapeutic need. Heart 2018;104(4):284-92.

10. Lanza GA, De Vita A, Kaski JC. "Primary” microvascular angina: clinical characteristics, pathogenesis and management. Interv Cardiol Rev 2018;13(3):108-11.

11. Alroy S, Preis M, Barzilai M et al. Endothelial cell dysfunction in women with cardiac syndrome X and MTHFR C677T mutation. Isr Med Assoc J 2007;9(4):321-5.

12. Seery JP. Therapeutic approach to microvascular angina (syndrome x). J Am Coll Cardiol 1995;25(6):1472.

13. O’Rourke MF, Nichols WW. Microvascular angina or "vis a tergo." J Am Coll Cardiol 2010;55(6):611.

14. Gould KL, Johnson NP. Coronary physiology beyond coronary flow reserve in microvascular angina: jacc state-of-the-art review. J Am Coll Cardiol 2018;72(21):2642-62.

15. Kaski JC, Russo G. Cardiac syndrome X: an overview. Hosp Pract 2000;35(2):75-94.
16. Khera R, Secemsky E, Wang Y et al. Revascularization practices and outcomes in patients with multivessel coronary artery disease who presented with acute myocardial infarction and cardiogenic shock in the us, 2009-2018. JAMA Intern Med 2020;180(10):1317-27.

17. Berry C, Sidik N, Pereira AC et al. Small-vessel disease in the heart and brain: current knowledge, unmet therapeutic need, and future directions. J Am Heart Assoc 2019;8(3):e011104.

18. Rahman H, Demir OM, Khan F et al. Physiological stratification of patients with angina due to coronary microvascular dysfunction. J Am Coll Cardiol 2020;75(20):2538-49.

19. Pelletier-Galarneau M, Dilsizian V. Microvascular angina diagnosed by absolute PET myocardial blood flow quantification. Curr Cardiol Rep 2020;22(2):9.

20. Manfrini O, Amaduzzi P, Bergami M et al. Effects of statin treatment on patients with angina and normal or nearly normal angiograms. Eur Cardiol 2020;15:e15. 